\title{
Hitomi X-ray Astronomy Satellite: Power of High-Resolution Spectroscopy
}

\author{
Hirokazu Odaka and the Hitomi Collaboration \\ Kavli Institute for Particle Astrophysics and Cosmology, \\ Stanford University, 2575 Sand Hill Rd, Menlo Park, CA 94026, USA \\ email: hodaka@stanford.edu
}

\begin{abstract}
Hitomi (ASTRO-H) is an X-ray observatory developed by an international collaboration led by JAXA. An X-ray microcalorimeter onboard this satellite has opened a new window of high-resolution spectroscopy with an unprecedented energy resolution of $5 \mathrm{eV}$ (FWHM) at $6 \mathrm{keV}$. The spacecraft was launched on February 17, 2016 from Tanegashima Island, Japan, and we completed initial operations including deployment of the hard X-ray imagers on the extensible optical bench. All scientific instruments had successfully worked until the sudden loss of the mission on March 26. We have obtained a spectrum showing fully resolved emission lines through the first-light observation of the Perseus Cluster. The line-of-sight velocity dispersion of $164 \pm 10 \mathrm{~km} \mathrm{~s}^{-1}$ reveals the quiescent environment of intracluster medium at the cluster core, implying that measured cluster mass requires little correction for the turbulent pressure. We also discuss observations to the Galactic Center which could be performed with Hitomi.
\end{abstract}

Keywords. space vehicles: instruments, techniques: spectroscopic, X-rays: galaxies: clusters, Galaxy: center, scattering

\section{Introduction}

X-rays have been important probes of extremely hot and energetic phenomena in the Universe among different scales from stellar-mass black holes to supermassive black holes in active galaxies, and to clusters of galaxies. Cluster of galaxies are the largest gravitationally bound objects in the Universe, and their deep gravitational potentials dominantly due to dark matter maintain plasma with temperature of $10^{7}-10^{8} \mathrm{~K}$, which can be best investigated with X-rays. The clusters are not static; dynamical motion is generated by accretion of gas and merging of clusters. Outflows from black holes are also thought to play essential roles in the cosmic evolution. Generally, they are highly ionized by strong X-ray radiation from their central engines (accretion disks or coronae), and thus display atomic line features in the X-ray band. High-resolution spectroscopy enables us to measure physical conditions including dynamics of these cosmic objects.

A scientific goal of the Hitomi mission (also known as ASTRO-H) was to study the structure and evolution of the Universe and physics in extreme conditions such as strong gravitation, high magnetic field, extreme density (e.g., super-nuclear density in neutron stars), and particle acceleration at shock waves. The Soft X-ray Spectrometer (SXS) onboard Hitomi, which is the first X-ray microcalorimeter pointed to a celestial source, significantly improved a spectral resolution to extended sources such as galaxy clusters and supernova remnants. The SXS also has the best spectroscopic performance for pointlike sources at iron K-shell band around $6 \mathrm{keV}$ while grating spectrometers, which are effective only for point-like sources, already opened a window of high-resolution spectroscopy at a softer band (typically below $3 \mathrm{keV}$ ). Another remarkable feature of the mission is its broadband coverage of $0.3-600 \mathrm{keV}$ by combination of four types of instruments. This simultaneous broadband measurement in the hard X-ray band above $10 \mathrm{keV}$ 
Table 1. Facts and figures of the four instruments

\begin{tabular}{|c|c|c|c|c|}
\hline & $\begin{array}{l}\text { Soft X-ray } \\
\text { Spectrometer } \\
\text { (SXS) }\end{array}$ & $\begin{array}{l}\text { Soft X-ray } \\
\text { Imager } \\
\text { (SXI) }\end{array}$ & $\begin{array}{l}\text { Hard X-ray } \\
\text { Imager } \\
\text { (HXI) }\end{array}$ & $\begin{array}{l}\text { Soft Gamma-ray } \\
\text { Detector } \\
\text { (SGD) }\end{array}$ \\
\hline $\begin{array}{l}\text { Detector } \\
\text { Technology }\end{array}$ & Micro-calorimeter & $\mathrm{CCD}$ & $\begin{array}{l}\mathrm{Si} / \mathrm{CdTe} \\
\text { cross-strips }\end{array}$ & $\begin{array}{c}\mathrm{Si} / \mathrm{CdTe} \\
\text { Compton camera }\end{array}$ \\
\hline Focal length & $5.6 \mathrm{~m}$ & $5.6 \mathrm{~m}$ & $12 \mathrm{~m}$ & $\mathrm{~N} / \mathrm{A}$ \\
\hline Effective area & $\begin{array}{l}300 \mathrm{~cm}^{2} \text { at } 6 \mathrm{keV} \\
250 \mathrm{~cm}^{2} \text { at } 1 \mathrm{keV}\end{array}$ & $\begin{array}{l}350 \mathrm{~cm}^{2} \text { at } 6 \mathrm{keV} \\
370 \mathrm{~cm}^{2} \text { at } 1 \mathrm{keV}\end{array}$ & $300 \mathrm{~cm}^{2}$ at $30 \mathrm{keV}$ & $\begin{aligned}> & 20 \mathrm{~cm}^{2} \text { at } 100 \mathrm{keV} \\
& (\text { Compton mode })\end{aligned}$ \\
\hline Energy range & $0.3-12 \mathrm{keV}$ & $0.4-12 \mathrm{keV}$ & $5-80 \mathrm{keV}$ & $60-600 \mathrm{keV}$ \\
\hline $\begin{array}{l}\text { Energy resolution } \\
(\text { FWHM) }\end{array}$ & $\begin{array}{l}<7 \mathrm{eV} \\
\text { at } 6 \mathrm{keV}\end{array}$ & $\begin{array}{l}<200 \mathrm{eV} \\
\text { at } 6 \mathrm{keV}\end{array}$ & $\begin{array}{l}<2 \mathrm{keV} \\
\text { at } 60 \mathrm{keV}\end{array}$ & $\begin{array}{l}<4 \mathrm{keV} \\
\text { at } 60 \mathrm{keV}\end{array}$ \\
\hline Angular resolution & $\sim 1.2^{\prime}$ & $\sim 1.3^{\prime}$ & $1.9^{\prime}$ & $\mathrm{N} / \mathrm{A}$ \\
\hline $\begin{array}{l}\text { Effective } \\
\text { field of view }\end{array}$ & $\begin{array}{c}3 \times 3 \\
\operatorname{arcmin}^{2}\end{array}$ & $\begin{array}{c}9 \times 9 \\
\operatorname{arcmin}^{2}\end{array}$ & $\begin{array}{c}38 \times 38 \\
\operatorname{arcmin}^{2}\end{array}$ & $\begin{array}{l}0.6 \times 0.6 \\
\text { degree }^{2}\end{array}$ \\
\hline Time resolution & $5 \mu \mathrm{s}$ & $4 \mathrm{~s} / 0.1 \mathrm{~s}$ & $25.6 \mu \mathrm{s}$ & $25.6 \mu \mathrm{s}$ \\
\hline Operating temperature & $50 \mathrm{mK}$ & $-120^{\circ} \mathrm{C}$ & $-20^{\circ} \mathrm{C}$ & $-20^{\circ} \mathrm{C}$ \\
\hline
\end{tabular}

is particularly important when soft X-ray features are results of reprocessing of intrinsic hard X-rays from accretion-powered central engines (e.g., black holes binaries and active galactic nuclei).

Hitomi is the sixth Japanese X-ray astronomy satellite as a successor to Suzaku. The observatory had been developed by a large international collaboration including Japan, USA, Canada, and Europe. The spacecraft was successfully launched by JAXA's H-IIA rocket from Tanegashima Island, Japan, on February 17, 2016, and we completed the initial critical phase which includes checkout of a cooling system of the microcalorimeter and deployment of the Extensible Optical Bench (EOB). However, the spacecraft lost its communication with ground stations on March 26, 2016, and separation of objects from the spacecraft and its rapid spin were observed. On April 28, JAXA announced that they decided to discontinue recovery efforts. Although planned observations could not be conducted, we obtained valuable data both from technical and scientific viewpoints during a month of the commissioning phase. The first-light observation focused on the Perseus Cluster, which is the X-ray brightest galaxy cluster. A spectrum of the core of the cluster obtained with the SXS clearly reveals quiescent environment in the cluster core, producing a certain impression of power of the high-resolution X-ray spectroscopy.

As well as clusters of galaxies and black holes, high-energy phenomena which emit strong X-rays seen in the central region of our Galaxy commands great interest. This means that the Galactic Center (GC) should be an important target of Hitomi. We also discuss what could be done with high-resolution spectroscopy toward science of the GC region.

\section{Mission Overview and Performance of the Microcalorimeter}

The Hitomi observatory has four types of instruments: the Soft X-ray Spectrometer (SXS), the Soft X-ray Imager (SXI), the Hard X-ray Imager (HXI), and the Soft Gammaray Detector (SGD) (Takahashi et al. 2016). Table 1 summarizes basic parameters of these instruments, and each position is drawn in Fig. 1. There are four focusing telescopes: two Soft X-ray Telescopes (SXTs) with focal lengths of $5.6 \mathrm{~m}$ are for the SXS and SXI, and two Hard X-ray Telescopes (HXTs) with focal lengths of $12 \mathrm{~m}$ are for two identical units 

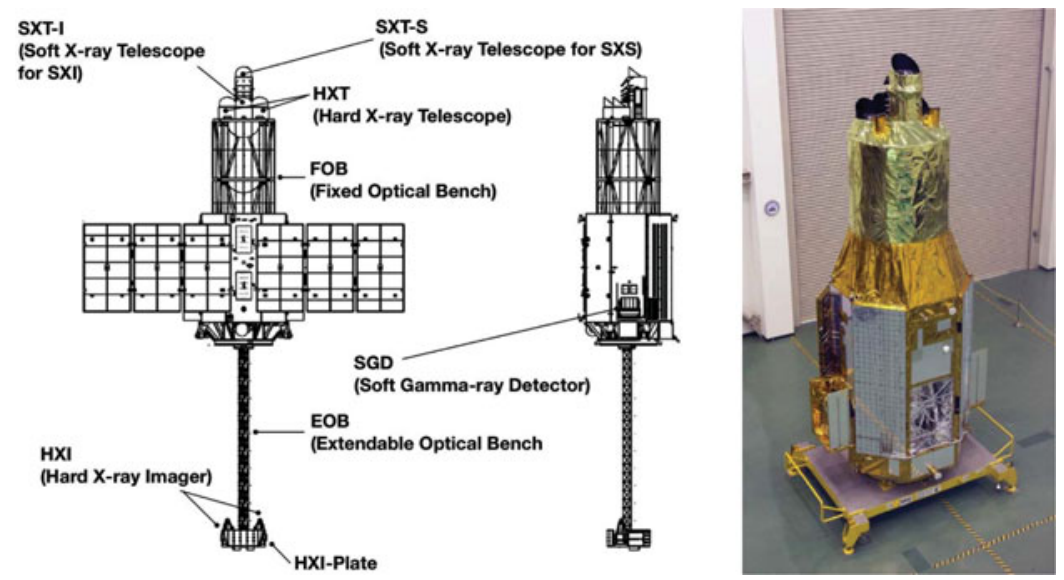

Figure 1. Structure of the Hitomi spacecraft showing the positions of the scientific instruments. The total length is $14 \mathrm{~m}$ after the EOB deployment in orbit. A photograph of the spacecraft is shown in the right panel. (Takahashi et al. 2016)
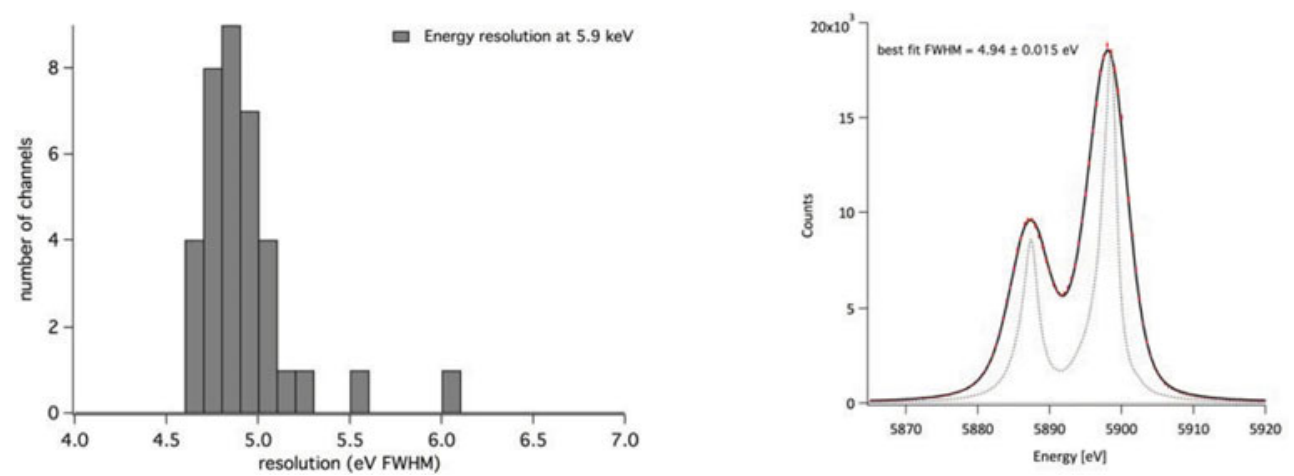

Figure 2. Left: histogram of energy resolutions measured in orbit using a ${ }^{55} \mathrm{Fe}$ calibration source from different microcalorimter pixels at $5.9 \mathrm{keV}$. Right: a whole-array spectrum of the $5.9 \mathrm{keV}$ doublet lines. The dotted line shows a line profile with natural broadening, and the solid line is fitted to the data points with the line model to which a Gaussian convolution is applied. (Takahashi et al. 2016)
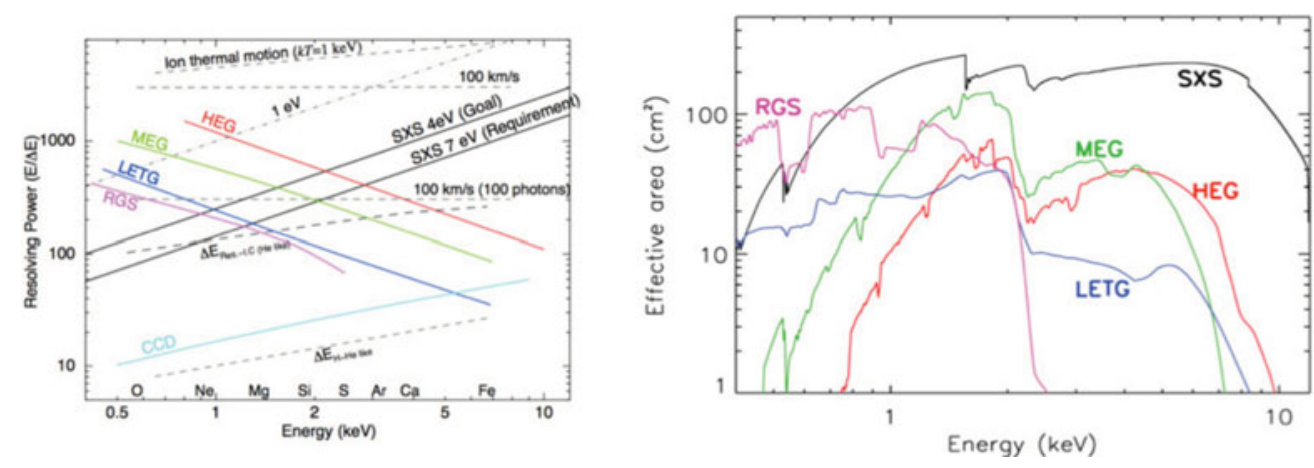

Figure 3. Resolving power $E / \Delta E$ (FWHM) (left) and effective area (right) of Hitomi-SXS and other existing instruments as functions of photon energy for comparison of their performance. 
of the HXI. The two units of the HXI, as focal plane detectors of the HXTs, are located at the end of the EOB, which meets the focusing condition after its deployment successfully conducted in the critical phase on February 28. After the extension, the total length of the spacecraft becomes $14 \mathrm{~m}$. The four different instruments simultaneously observe a celestial source, covering a broad energy band from $0.3 \mathrm{keV}$ up to $600 \mathrm{keV}$. The two soft X-ray instruments measure photons of $0.3-12 \mathrm{keV}$, and the HXT-HXI system focuses hard X-rays between $5 \mathrm{keV}$ and $80 \mathrm{keV}$. The SGD covers the highest energy band up to $600 \mathrm{keV}$ by semiconductor Compton camera technology, which is the only instrument that does not use a focusing telescope.

The spacecraft was launched by 30th H-IIA launch vehicle from Tanagashima Space Center, JAXA on February 17, 2016, and put into an approximately circular low-Earth orbit with an altitude of $575 \mathrm{~km}$ and an inclination of $31^{\circ}$. The launch operations went smoothly and the orbit was exact as planned. After the initial operations including checkout of the spacecraft bus functions (e.g., communication, attitude control) and start-up of the SXS cooler, JAXA succeeded in deployment of the EOB where the two units of the HXI are mounted. We observed several X-ray sources: the Perseus cluster (February 25-27, March 4-8), N132D (March 8-11), IGR J16318-4848 (March 11-15), RXJ 18563754 (March 17-19, 23-25), G21.5-0.9 (March 19-23), and the Crab Nebula (March 25). A main purpose of these observations was calibration of the instruments, but they also provided us with valuable scientific data. The SXS was already turned on at the observation of the Perseus cluster, and the SXI started observation during the pointing to Perseus. We completed start-up operations of all the instruments before the loss of contact. As described in the introduction section, the satellite was lost on March 26. Detailed technical mechanisms of the incident was reported by JAXA (JAXA's report 2016).

We summarize here the system overview and initial in-orbit performance of the microcalorimeter (SXS). For other instruments, see reports by the Hitomi Collaboration (mission overview: Takahashi et al. 2016, SXS: Kelley et al. 2016, SXI: Tsunemi et al. 2016, HXI: Nakazawa et al. 2016, SGD: Watanabe et al. 2016, SXT: Okajima et al. 2016, HXT: Awaki et al. 2016) and subsequent journaled papers. The SXS has $6 \times 6$ pixels which cover a field of view of $3^{\prime} \times 3^{\prime}$. The microcalorimeter pixel array has to be cooled down to $50 \mathrm{mK}$ with a chain of coolers composed of a three-stage adiabatic demagnetization refrigerator, superfluid liquid ${ }^{4} \mathrm{He}$, a ${ }^{4} \mathrm{He}$ Joule-Thomson cryocooler, and two-stage Stirling cryocoolers. A helium vent valve was opened to establish the liquid helium pumping soon after the rocket fairing was opened. Then, we started turning-on operations of the cooler chain, the sensor reached to $50 \mathrm{mK}$ on the 5 th day after the launch, and the cooling system continued to work without any problem until the end of the mission. In-orbit measurement of a calibration X-ray source of ${ }^{55} \mathrm{Fe}$ confirmed that the sensor achieved an energy resolution $4.9 \mathrm{eV}$ (FWHM) at $5.9 \mathrm{keV}$. Fig. 2 shows a histogram of the measured energy resolutions and the measured spectrum by using the calibration source. We make comparison with other existing instruments in Fig. 3. Even for point-like sources the microcalorimeter has better spectral resolution above $\sim 2 \mathrm{keV}$, and moreover the SXS has an order of magnitude larger effective area at iron K-shell band around $6 \mathrm{keV}$.

\section{Initial Results from the First Light: the Perseus Cluster}

Clusters of galaxies are important probes of cosmological parameters and large-scale astrophysical processes as they are the largest gravitationally bound objects in the universe (Allen, Evrard, \& Mantz 2011, Kitayama et al. 2014). They contains hot diffuse 

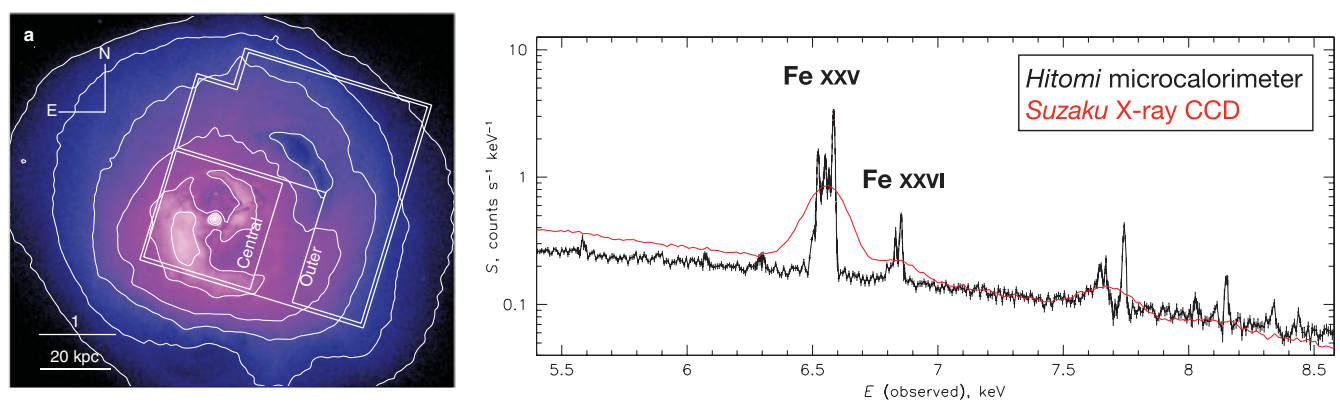

Figure 4. Left: an X-ray image of the Perseus cluster obtained with Chandra and the spectrum extraction region of Hitomi. Right: a first-light spectrum of the Perseus cluster using the whole arrays of the SXS. Fe XXV He $\alpha(6.6 \mathrm{keV})$ and Fe XXVI Ly $\alpha(6.8 \mathrm{keV})$ are clearly resolved into each atomic transitions. The Suzaku-CCD spectrum, which had the best spectral resolution for extended sources before Hitomi, is superposed. Its different slope of the continuum is due to a different detector response. (Hitomi Collaboration 2016a)

gas with temperature of 50 million kelvin as the dominant baryonic component, which is only seen in the X-ray band. Particularly, measurement of turbulence is essential to understand effects of central supermassive black holes (Boehringer et al. 1993, Churazov et al. 2011, Fabian 2012), and to obtain accurate masses of clusters. In the very initial phase, Hitomi observed the Perseus cluster, which is the X-ray brightest galaxy cluster, for an exposure time of 230 thousand seconds (Hitomi Collaboration 2016a). This target was selected for the first demonstration of high-resolution spectroscopy.

The first analysis of the spectrum around iron K-shell complex (5.5-8.5 keV) is shown in the right panel of Fig. 4 while the left panel shows the spectrum extraction region superposed on a fine-resolution X-ray image obtained with Chandra. In the spectrum, we also show a spectrum obtained with X-ray CCD onboard Suzaku, which had the best spectral resolution for extended sources before the launch of Hitomi. The lines are clearly resolved, revealing that the core of the cluster is less turbulent than we expected. The lineof-sight velocity dispersion is determined to be $164 \pm 10 \mathrm{~km} \mathrm{~s}^{-1}$. The turbulent pressure support is only $4 \%$ of thermal pressure, which implies that a total cluster mass determined by hydrostatic equilibrium would require little correction for this turbulent pressure. More complete confirmation of this assumption should be done by future extended observations with a microcalorimeter covering outer regions and other clusters. From an astrophysical viewpoint, the quiescent intracluster medium is a surprise since the central supermassive black hole has been thought to introduce turbulent via its energetic relativistic jet.

The high-energy resolution of the SXS (and the low turbulent velocity) enables us to resolve He $\alpha$ lines of Fe XXV at $6.5 \mathrm{keV}$ in the observing frame into four individual transitions: $\mathrm{w}$ (resonance): $1 s 2 p^{1} \mathrm{P}_{1} \rightarrow 1 s^{2}{ }^{1} \mathrm{~S}_{0}, \mathrm{x}$ (intercombination): $1 s 2 p^{3} \mathrm{P}_{2} \rightarrow 1 s^{2}{ }^{1} \mathrm{~S}_{0}, \mathrm{y}$ (intercombination): $1 s 2 p^{3} \mathrm{P}_{1} \rightarrow 1 s^{2}{ }^{1} \mathrm{~S}_{0}$, and $\mathrm{z}$ (forbidden): $1 s 2 s^{3} \mathrm{P}_{1} \rightarrow 1 s^{2}{ }^{1} \mathrm{~S}_{0}$. Intensity ratios between these lines can be used for diagnostics of temperature and density. We find a signature that the resonance $(\mathrm{w})$ line is slightly suppressed compared with theoretical models that assume optically thin plasma under collisional ionization equilibrium (Foster et al. 2011, Kaastra et al. 1996). This suggests that resonance scattering is effective at the core of the cluster (Zhuravleva et al. 2013). Another energy band of interest band is around $3.5 \mathrm{keV}$ where several authors reported an unknown line which can be interpreted as an annihilation signal of sterile neutrino. However, we do not detect any line feature in this energy region from the first light observation of the Perseus cluster (Hitomi Collaboration 2016b). 

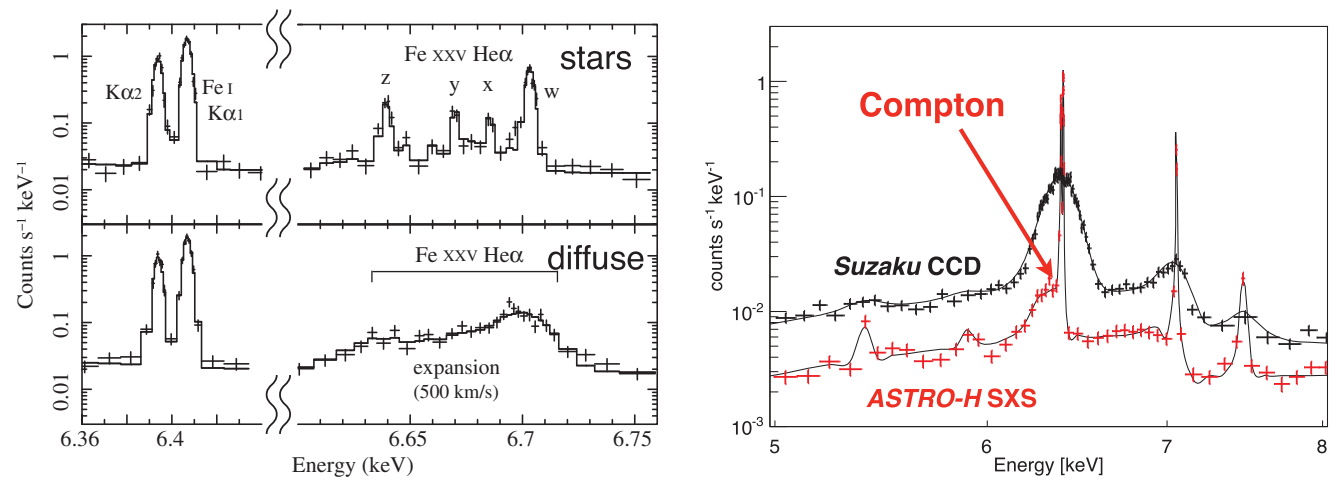

Figure 5. Left: simulations (exposure time: $100 \mathrm{ks}$ ) of the SXS spectrum for two different origins: (1) plasma is associated with stars (accreting white dwarfs and magnetically active stars) and (2) truly diffuse interstellar plasma is expanding at a velocity of $500 \mathrm{~km} \mathrm{~s}^{-1}$ (Koyama et al. 2014). Right: detailed Monte-Carlo simulations of the Compton shoulder profile emerging from the Sgr B2 cloud convolved with detector spectral response functions of Hitomi-SXS and Suzaku-XIS where we assume an exposure time of $200 \mathrm{ks}$ (Odaka et al. 2011).

\section{Potential Prospects for the Galactic Center}

X-rays bring us important information on energetic activities at the center of our Galaxy (Koyama et al. 2014). The X-ray emission seen in the GC region is characterized by a mixture of emissions from highly ionized plasma and fluorescent X-rays from cold matter. The plasma is sufficiently hot to emit emission lines of hydrogen-like ion of iron (Fe XXVI), and thus is impossible to be confined by gravitation if the plasma is truly diffused in the GC region. The nature of the extended hot plasma is still mysterious: an interstellar diffuse plasma or integrated emission of a number of point-like sources. The fluorescent emission, on the other hand, is interpreted as reflection of past flares of the central black hole Sgr $A^{*}$ by cold giant molecular clouds. Thus, the reflection features should be important targets for investigating the past activities of Sgr A*. The reflecting clouds are probes of flares with luminosities about $10^{39} \mathrm{erg} \mathrm{s}^{-1}$ and time within $\sim 1000$ years. An evidence of a much larger but older outburst, which is believed to have produced the Fermi bubble, could be found as X-ray relics of diffuse plasma. In addition, search for signals of dark matter is another important problem.

An X-ray microcalorimeter may distinguish the two different possible origins of the hot extended plasma. Although the Galactic ridge X-ray emission is resolved into a number of point sources with Chandra (Revnivtesv et al. 2009), the Galactic center emission seems to be dominated by a different component whose origin is unknown (Uchiyama et al. 2013). Since the gravitation potential is not able to confine the truly diffuse plasma of that temperature, the hot plasma would be highly likely to expand at high velocity of $\sim 500 \mathrm{~km} \mathrm{~s}^{-1}$. The left panel of Fig. 5 shows simulations of the SXS spectrum around helium-like iron recombination lines $(6.7 \mathrm{keV})$ and neutral iron fluorescence lines (6.4 $\mathrm{keV}$ ) for two different possible situations: (1) plasma is associated with stars (accreting white dwarfs and magnetically active stars) and (2) truly diffused interstellar plasma is expanding at a velocity of $500 \mathrm{~km} \mathrm{~s}^{-1}$. We assume that plasma associated with stars does not have high-velocity component.

A new spectral feature called Compton shoulder will be useful in the era of microcalorimeters. The Compton shoulder is a tail-like structure with the iron $\mathrm{K} \alpha$ line at $6.4 \mathrm{keV}$ generated by Compton down-scattering in a dense cloud. This is a new spectral probe of geometry, column density, metal abundance, and time variability (Sunyev 
\& Churazov 1998, Matt 2002, Odaka et al. 2011, Odaka et al. 2016). The right panel of Fig. 5 shows detailed Monte-Carlo simulations of the Compton shoulder profile emerging from the Sgr B2 cloud convolved with detector spectral response functions of Hitomi-SXS and Suzaku-XIS. The Compton shoulder is too small to be revolved even with the best resolution achieved by an X-ray CCD. The SXS would be able to measure the profile of the shoulder. Since the reflection emission by Sgr B2 is getting faint (Nobukawa et al. 2009, Zhang et al. 2015) X-ray reflecting clouds in the vicinity of Sgr A (Ponti et al. 2010) would be better targets in the near future.

\section{Summary}

The X-ray microcalorimeter (SXS) onboard Hitomi was successfully operated at 50 milli-kelvin, and the entire SXS system worked perfectly. The sensor achieved an unprecedented spectral resolution of $4.9 \mathrm{eV}$ (FWHM) at $6 \mathrm{keV}$, or resolving power of $E / \Delta E=1250$. Other scientific instruments (SXI, HXI, SGD) and telescopes (SXTs, HXTs) also worked well. We conducted the first light observation pointed to the Perseus cluster and revealed that the core of the cluster is less turbulent than expected through measurement of the narrow emission lines of highly ionized iron. Although the satellite was lost, it showed the power of the high-resolution X-ray spectroscopy, and would also be promising for studying energetic activities, structure and history of the Galactic center region.

\section{References}

Awaki, H., et al. 2016, Proc. of SPIE, 9905, 35

Allen, S. W., Evrard, A. E., \& Mantz, A. B. 2011, Annu. Rev. Astron. Astrophys. 49, 409

Boehringer, H., et al. 1993, MNRAS 264, L25

Churazov, E., Forman, W., Jones, C., \& Böhringer, H. 2000, A\&ऽA 356, 788

Fabian, A. C. 2012, Annu. Rev. Astron. Astrophys. 50, 455

Foster, A. R., Li, J., Smith, R. K., \& Brickhouse, N. S. 2011, ApJ 756, 128

Hitomi Collaboration 2016a, Nature, 535, 117

Hitomi Collaboration 2016b, ApJL, submitted

Kaastra, J. S., Mewe, R., \& Nieuwenhuijzen, H. 1996, 11th Colloquium on UV and X-ray Spectroscopy of Astrophysical and Laboratory Plasmas 411

Kelley, R. L., et al. 2016, Proc. of SPIE, 9905, 28

Kitayama, T. et al. 2014, ASTRO-H White Paper: Clusters of Galaxies, arXiv:1412.1176

Koyama, K. et al. 2014, ASTRO-H White Paper: Galactic Center, arXiv:1412.1170

Matt, G. 2002, MNRAS, 337, 147

Nakazawa, K. et al. 2016, Proc. of SPIE, 9905, 34

Nobukawa, M. et al. 2011, ApJL, 739, L52

Okajima, T., et al. 2016, Proc. of SPIE, 9905, 32

Odaka, H. et al. 2011, ApJ, 740, 103

Odaka, H., Yoneda, H., Takahashi, T., \& Fabian, A. C. 2016, MNRAS, in press

Ponti, G. et al. 2010, ApJ, 714, 732

Revnivtesv, M. et al. 2009, Nature, Nature, 458, 1142

Sunyaev R. \& Churazov E. 1998, MNRAS, 297, 1279

Takahashi, T. et al. 2016, Proc. of SPIE, 9905, 27

Tsunemi, H., et al. 2016, Proc. of SPIE, 9905, 33

Uchiyama, H. et al. 2013, PASJ, 65, 19

Watanabe, S., et al. 2016, Proc. of SPIE, 9905, 36

Zhang S. et al. 2015, ApJ, 815, 132

Zhuravleva, I. et al. 2013, MNRAS, 435, 3111 